\title{
A Silent Closed Rupture of the Flexor Digitorum Profundus on the Index Finger: An Unsolved Mystery
}

\author{
Hyun-Dong Yeo (D), Na-Hyun Hwang (D), Seung-Ha Park (D), Byung-II Lee (D), Eul-Sik Yoon (D), Jae-Ho Chung (D) \\ Department of Plastic and Reconstructive Surgery, Korea University Anam Hospital, Korea University College of Medicine, Seoul, Korea
}

\begin{abstract}
We report the case of a patient who fully recovered from a closed flexor tendon rupture through a two-stage flexor tendon reconstruction using silicone rods, despite a considerable delay in treatment. A 17-year-old male patient visited our clinic with a sudden inability to flex his left index finger, although there were no signs of injury. Magnetic resonance imaging revealed an extensive rupture of the flexor digitorum profundus from the base of the distal phalanx to the proximal phalangeal joint of his left hand. After a two-stage tendon graft operation was performed, the patient regained full flexion of the index finger and was able to hold a fist without any limitations in range of motion. Complete rupture of tendons usually accompanies history of trauma or underlying tendon pathology. In our case, however, the tendon rupture occurred silently with no obvious underlying causes. It is important to recognize the signs to evaluate the underlying structures for appropriate management and treatment. Even with considerable delay, the patient may regain full function of the tendon.
\end{abstract}

Keywords: Rupture; Tendons; Silicones; Reconstructive surgical procedures

\section{Introduction}

Flexor tendon ruptures usually present with loss of active flexion and often accompany open injuries. Closed tendon ruptures with delayed onset are therefore infrequent. In such rare cases, diagnosis and treatment may be significantly delayed, leading to long-term sequelae including poor outcome in range of motion [1]. Early diagnosis and treatment are therefore important for the best results.

We report a case of a patient who presented with a closed rupture of flexor digitorum profundus (FDP) of the index finger in zone II. The patient had no previous symptoms or signs of trauma, nor was there any evidence of underlying tendon pathology. Despite the considerable delay in treatment, the patient showed full restoration of tendon function after a two-stage flexor tendon reconstruction using silicone rods. This study was performed in accordance with the principles of the Declaration of Helsinki. Written informed consent was obtained from the patient.

\section{Case}

A 17-year-old male patient visited our clinic after accidentally putting his left ring finger into a ventilation fan. The patient showed a laceration injury on the dorsum of the left fourth metacarpophalangeal joint with no signs of further injuries on other digits. The ring finger was repaired and the patient was discharged with no major complica-

\section{Case Report}

Received: January 14, 2021

Revised: March 1, 2021

Accepted: March 3, 2021

\section{Corresponding author: \\ Na-Hyun Hwang, M.D., Ph.D.}

Department of Plastic and Reconstructive Surgery, Korea University Anam Hospital, 73 Goryeodae-ro, Seongbuk-gu, Seoul 02841, Korea

Tel: +82-2-920-5368

Fax: +82-2-922-7437

E-mail: prissy426@gmail.com

This is an Open Access article distributed under the terms of the Creative Commons Attribution Non-Commercial License (https://creativecommons.org/licenses/by-nc/4.0/) which permits unrestricted non-commercial use, distribution, and reproduction in any medium, provided the original work is properly cited.

(c) 2021 Korean Wound Management Society 
tions.

Six weeks later, the patient revisited the clinic with a sudden inability to flex the left index finger (Fig. 1). A physical exam demonstrated lack of index finger flexion. Magnetic resonance imaging (MRI) revealed an extensive rupture of the FDP from the middle phalangeal level to the proximal phalangeal joint of his left hand (Fig. 2).

Taking the delayed diagnosis and the nature of the injury into account, a two-stage tendon graft operation was planned.

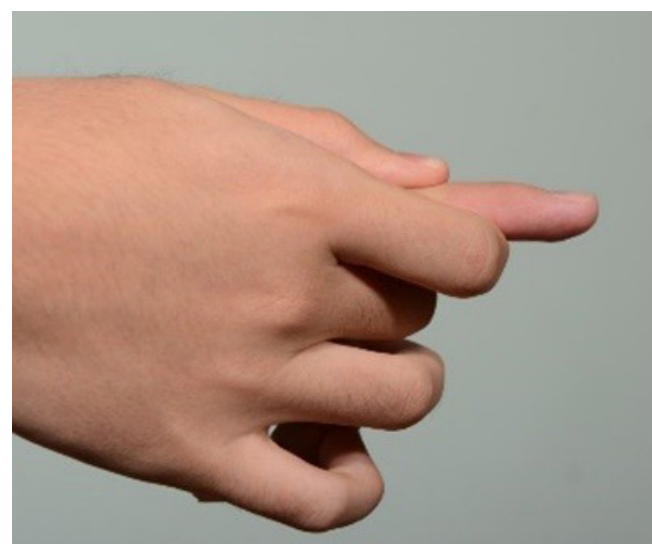

Fig. 1. Preoperative photograph of the left second finger.

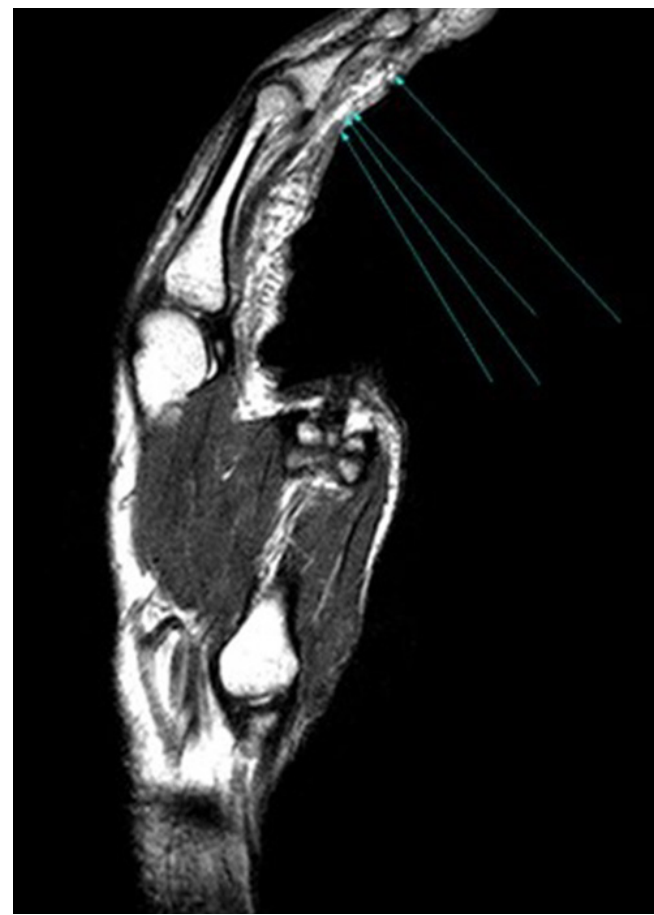

Fig. 2. Magnetic resonance imaging (MRI). MRI showing complete rupture of the second flexor digitorum profundus at the middle phalangeal level (blue arrows).
During the primary operation, a Hunter rod was inserted and secured to the proximal and distal portions of the ruptured tendon without further trimming (Fig. 3). The patient was able to flex the index finger after the initial silicone Hunter rod implant (Fig. 4). Three months later, the Hunter rod was removed and $3.5 \mathrm{~cm}$ of the palmaris longus tendon was harvested and transplanted (Fig. 5). For the first 4 weeks after stage II surgery, the patient was trained to perform passive flexion and active extension of the index finger using a rubber band traction. A rubber band was attached to a hook which was glued onto the edge of the nail and proximally to a safety pin attached around
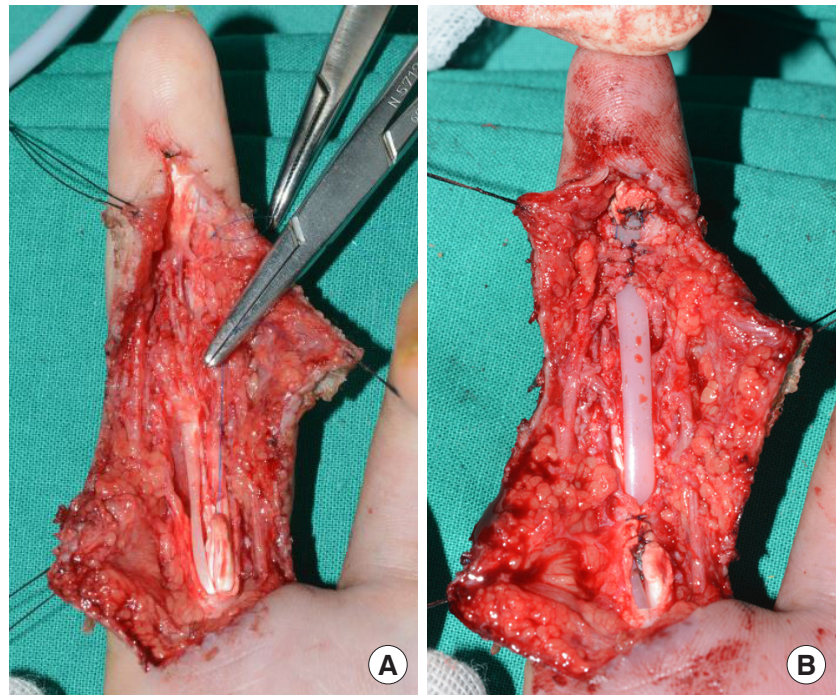

Fig. 3. Intraoperative photographs of stage I reconstruction. (A) Ruptured tendon. (B) Placement of a silicone rod between the distal and proximal ends of the ruptured tendon.

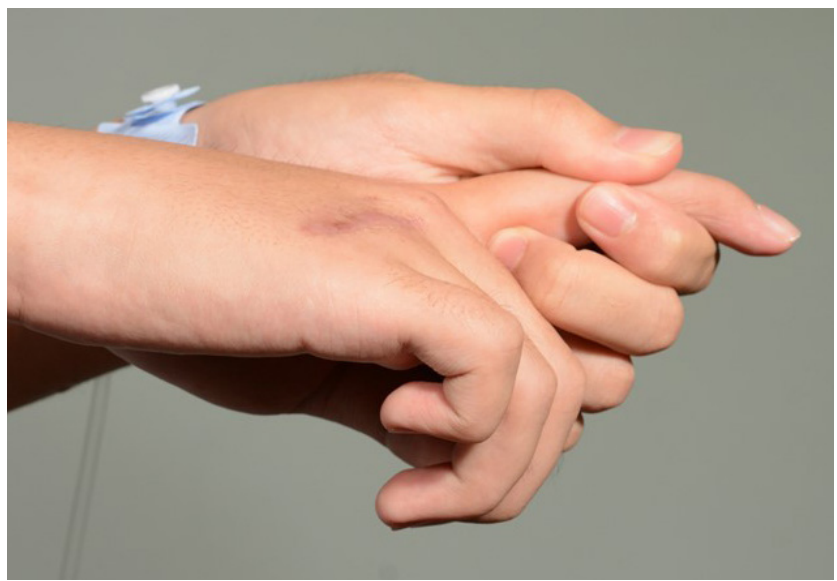

Fig. 4. Three-month postoperative photo. Patient is able to flex the index finger after stage I reconstruction. 

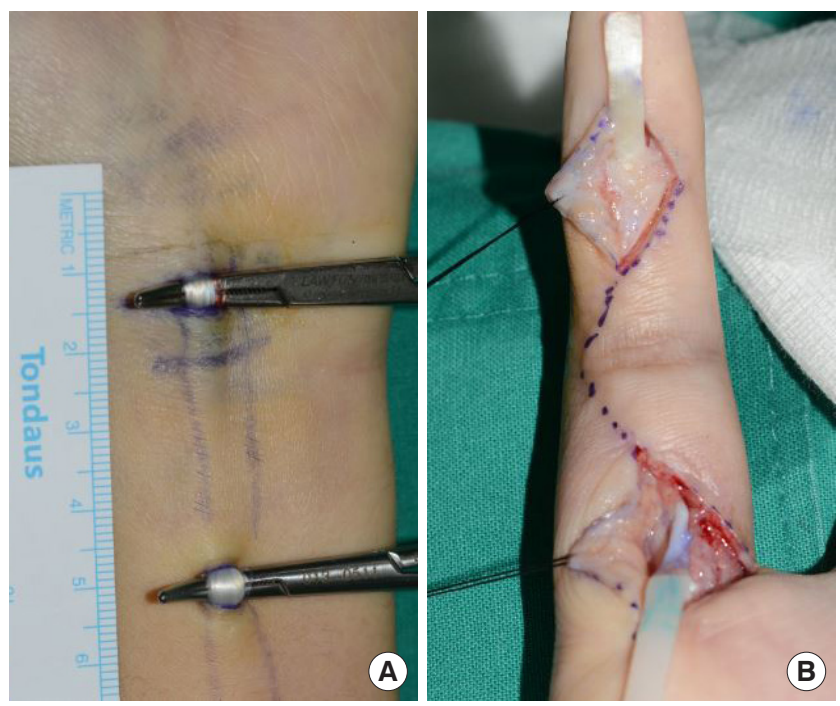

Fig. 5. Intraoperative photographs of stage II reconstruction. (A) The palmaris longus tendon was extracted. (B) The distal end of the implanted silicone rod and the extracted palmaris longus tendon was sutured and passed under the pulley to its distal end.

the distal palmar crease. From the 5th week, active flexion exercises were maintained for another 2 weeks followed by progressive resistance exercise. The splint was removed 6 weeks after surgery. As a result, the patient regained full flexion of the index finger and was able to make a fist without any limitations in range of motion (Fig. 6).

\section{Discussion}

Closed tendon ruptures are rare, but require surgical intervention. Unfortunately, unlike open tendon injuries, closed tendon ruptures are often not diagnosed on initial presentation. In such cases, reconstruction with primary tendon grafts usually fails due to scar tissue formation and contracture. A staged operation is often necessary to restore the flexor tendon bed by first inserting a flexible silicone prosthesis, followed by a secondary tendon graft 2 or 3 months later. Several studies on this two-stage tendon reconstruction method have been reported, but with varied results [2-4]. Ultimately, early recognition and meticulous technique can help ensure a successful stage flexor tendon reconstruction [5].

In our case, the delay was partly due to the asymptomatic, or "silent" nature of the injury. The rare closed rupture is likely due to underlying causes such as a fracture, cystic degeneration, rheumatoid arthritis, or sequelae following local steroid injection in mid-substance rupture. Compulsory digital extension during maximum active flexion can also cause a closed

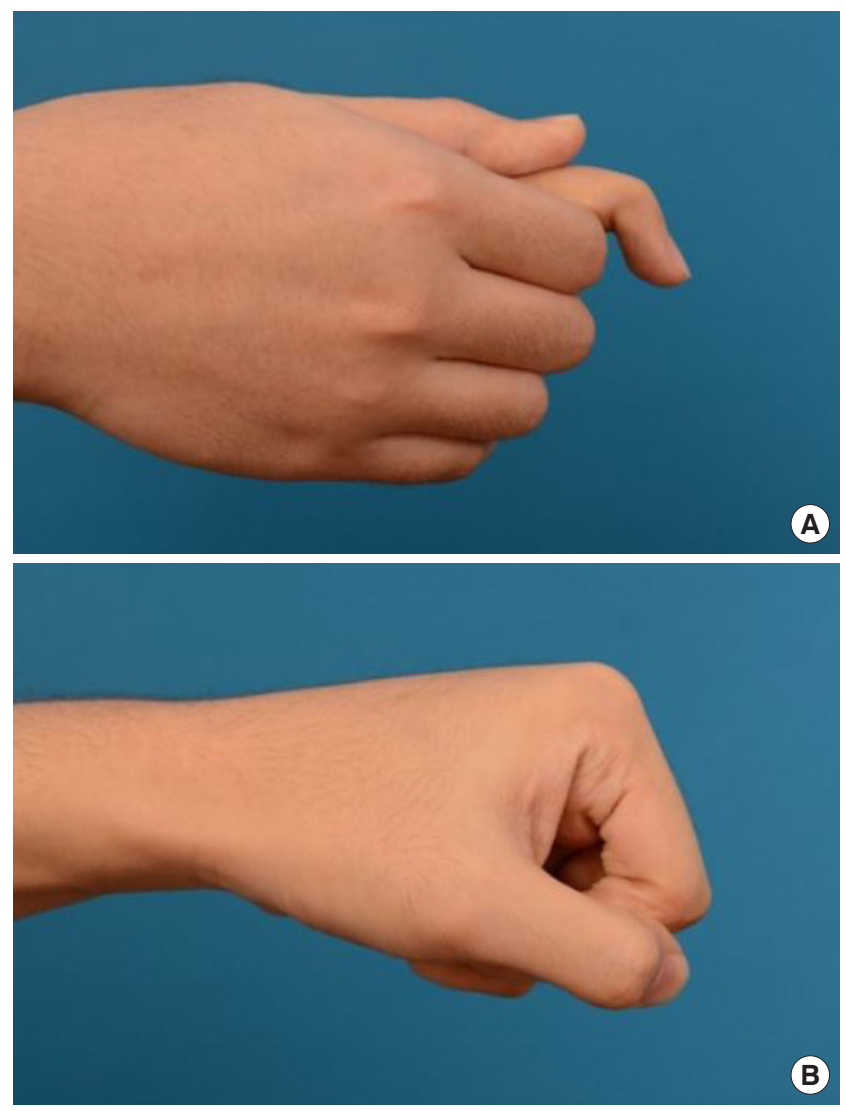

Fig. 6. One year and 5 months postoperative photographs. (A) Full flexion of the distal phalanx. (B) Full fist clenching.

tendon rupture. Our patient, however, did not have an obvious cause and neither was any underlying pathology noted. We also found that the ruptured site was in zone II of the index finger, whereas most reports show the site of rupture is almost exclusively around the tendon-bone insertion in zone I [6-10].

Most tendon ruptures present with open wounds around the level of the injury and additional surgical incisions are usually made by way of extending these existing wounds. However, in closed tendon ruptures the exact level of injury may be difficult to ascertain. Radiologic imaging such as MRI permits preoperative assessment of tendon status including the extent of any proximal retraction, enabling precise planning of surgery. Preoperative, accurate assessment of tendon pathology can minimize unnecessary dissection and simplify the operation [11].

Despite having been diagnosed long after the initial injury, our case showed full restoration of flexor tendon function. None of the complications that can occur after staged flexor tendon reconstruction, such as repaired tendon rupture, silicone prosthesis synovitis, infection, and adhesion, were ob- 
served [2]. Early active motion protocol is known to minimize adhesion formation, and the pseudo-sheath formed after stage I also reduces the risk of adhesion by providing a smooth tunnel for the tendon graft to glide through [12-14].

The exact cause of spontaneous tendon rupture is usually multifactorial, and microscopic pathology may be present in some cases. Folmar et al. [15] investigated flexor tendon ruptures in non-rheumatoid hands, and found that some cases seemed to be caused by intrinsic tendon pathology. Anatomical tendon differences were also reported as potential triggers of spontaneous ruptures, such as the bifurcation of the common FDP tendon to the ring and small finger [15]. Notwithstanding, our case demonstrated that even with delayed reconstruction, tendon functions may be fully restored with accurate assessment and treatment.

In conclusion, complete rupture of tendons usually accompany history of trauma or underlying tendon pathology. In our case, however, the tendon rupture occurred silently with no obvious underlying causes. It is important to recognize the signs to evaluate the underlying structures for appropriate management and treatment. Even with considerable delay, the patient may regain full function of the tendon.

\section{Conflict of interest}

No potential conflict of interest relevant to this article was reported.

\section{ORCID iDs}

Hyun-Dong Yeo

Na-Hyun Hwang

Seung-Ha Park

Byung-Il Lee

Eul-Sik Yoon

Jae-Ho Chung

\section{References}

1. Stamos BD, Leddy JP. Closed flexor tendon disruption in athletes. Hand Clin 2000;16:359-65.

2. Ahmad T, Mohsin M, Zaroo MI, et al. Two stage flexor tendon reconstruction in hand: our experience. Int J Res
Med Sci 2016;4:4697-700.

3. Unglaub F, Bultmann C, Reiter A, et al. Two-staged reconstruction of the flexor pollicis longus tendon. J Hand Surg Br 2006;31:432-5.

4. Smith P, Jones M, Grobbelaar A. Two-stage grafting of flexor tendons: results after mobilisation by controlled early active movement. Scand J Plast Reconstr Surg Hand Surg 2004;38:220-7.

5. Soucacos PN, Beris AE, Malizos KN, et al. Two-stage treatment of flexor tendon ruptures: silicon rod complications analyzed in 109 digits. Acta Orthop Scand Suppl 1997;275: 48-51.

6. Fukui A, Kido A, Inada Y, et al. Closed rupture of the flexor digitorum profundus tendon of the little finger caused by calcification of the triangular-fibrocartilage. J Hand Surg Br 1996;21:375-7.

7. Hartford JM, Murphy JM. Flexor digitorum profundus rupture of the small finger secondary to nonunion of the hook of hamate: a case report. J Hand Surg Am 1996;21: 621-3.

8. Prosser GH, Sterne GD, Nancarrow JD. Intratendinous rupture of flexor digitorum profundus caused by non-specific synovitis. Br J Plast Surg 2002;55:77-9.

9. Simman R, Fietti VG. Closed rupture of the flexor digitorum profundus tendon of the left little finger. Hosp Physician 2000;36:55-9.

10. Netscher DT, Badal JJ. Closed flexor tendon ruptures. J Hand Surg Am 2014;39:2315-23.

11. Agarwal P. Closed rupture of the flexor digitorum profundus tendon of little finger: a case report. Indian J Plastic Surg 2004;37:71-3.

12. Amadio PC, Wood MB, Cooney WP 3rd, et al. Staged flexor tendon reconstruction in the fingers and hand. J Hand Surg Am 1988;13:559-62.

13. Khanna A, Friel M, Gougoulias N, et al. Prevention of adhesions in surgery of the flexor tendons of the hand: what is the evidence? Br Med Bull 2009;90:85-109.

14. Khanna A, Gougoulias N, Maffulli N. Modalities in prevention of flexor tendon adhesion in the hand: what have we achieved so far? Acta Orthop Belg 2009;75:433-44.

15. Folmar RC, Nelson CL, Phalen GS. Ruptures of the flexor tendons in hands of non-rheumatoid patients. J Bone Joint Surg Am 1972;54:579-84. 